\title{
Association of Primary Care Providers Health Habits with Lifestyle Counseling to Hypertensive Elderly Patient: Results of a National Survey
}

\author{
Ihab Hajjar ${ }^{1}$, Brandy Frost ${ }^{2}$, John E. Lacy ${ }^{2}$, Jane Kotchen ${ }^{3}$ \\ ${ }^{1}$ Hebrew SeniorLife and Harvard Medical School \\ ${ }^{2}$ University of South Carolina, School of Medicine \\ ${ }^{3}$ Medical College of Wisconsin
}

\begin{abstract}
We were interested in studying the association between personal health habits of healthcare providers and their beliefs and practices of lifestyle counseling to elderly hypertensive patients. We conducted a national anonymous cross sectional survey of physicians and nurse practitioners providing primary care. The survey was developed to assess providers' beliefs and practices of lifestyle counseling as well as personal habits. Beliefs about counseling were assessed by (a) perceived available time, (b) perceived skill, and (c) perceived effectiveness of lifestyle counseling. Reported lifestyle counseling practice were assessed by reported frequency of (a) obtaining lifestyle habits and (b) providing lifestyle counseling to elderly hypertensive patients. We mailed 7,500 mailed surveys and received 774. Providers who reported healthier lifestyle habits reported that they more frequently obtain lifestyle history $(\mathrm{p}=.004)$ and provide lifestyle counseling $(\mathrm{p}<.001)$. These providers also more frequently believed that counseling was effective $(<.001)$, that they had available time to provide counseling $(\mathrm{p}=.008)$, and that they were skilled in counseling $(\mathrm{p}<.001)$ independent of other factors. Providers with healthier habits reported more frequent lifestyle counseling and positive beliefs about counseling. Programs that would improve health habits in primary care providers may improve lifestyle counseling to elderly hypertensive patients.
\end{abstract}

(C) 2006 Californian Journal of Health Promotion. All rights reserved.

Keywords: Hypertension, elder health, counseling, physical activity

\section{Introduction}

The elderly population continues to carry the highest burden of hypertension and cardiovascular disease. In the US, although close to $65 \%$ of those 60 years or older have hypertension, only $27 \%$ were controlled in 1999 2000 to below 140/90 mm Hg (Hajjar, Miller, \& Hirth, 2002). Uncontrolled hypertension translates to increased cardiovascular events and mortality (Chobanian et al., 2003). Poor lifestyle, such as unhealthy diets, low levels of physical activity and obesity, contributes to this high hypertension burden. However, this burden is modifiable (Shah \& Cook, 2001).

Many clinical trials have documented the effectiveness of lifestyle counseling in improving patients' health behaviors (Calfas, Long, Sallis, Wooten, Pratt, Patrick, 1996;
Linden, Lenz, \& Con, 2001; Ockene et al., 1999; Petrella \& Lattanzio, 2002). Moreover, national and international guidelines recommend providing counseling to all patients with hypertension to improve lifestyle behavior (Chobanian et al., 2003; Greenlund, Giles, Keenan, Croft, \& Mensah, 2002; Hansson, Hedner, \& Himmelmann, 1999). However, many patients are not receiving adequate counseling from their health care providers (Fallon, Wilcox, \& Laken, 2006; Centers for Disease Control and Prevention, 1999). For example, according to the National Ambulatory Medical Care Survey 1995-2000, only 29\% of patients with hypertension had diet counseling and $20 \%$ had physical activity counseling (Collins, D'Angelo, Stearns, \& Campbell, 2005; Ma, Urizar, Alehegn, \& Stafford, 2004). Older patients were less likely to receive such 
counseling. In 1998, data from the National Health Interview Survey showed that close to half of patients with hypertension have not been counseled on losing weight or increasing physical activity to lower blood pressure (Egede \& Zheng, 2002).

We have previously reported that primary care providers' likelihood of counseling their hypertensive patients about lifestyle is negatively associated with the patient's age (Hajjar et al., 2002). We were interested in exploring factors that would predict providers' frequency of lifestyle counseling in elderly hypertensive patients. Evidence from prior surveys targeting overall patient counseling suggested that personal health behaviors of providers may be associated with lifestyle counseling to their respective patients (Abramson, Stein, Schaufele, Frates, \& Rogan, 2000; Frank, Breyan, \& Elon, 2000; Wells, Lewis, Leake, \& Ware, 1984). Whether this would also apply to counseling elderly hypertensive patients is not known. In addition, providers' positive beliefs and attitudes about counseling and prevention may predict the likelihood of providing counseling to their patients (Litaker, Flocke, Frolkis, \& Stange, 2005). We were therefore interested in exploring the association between personal lifestyle habits and the beliefs and attitudes towards lifestyle counseling.

Prior surveys that have attempted to investigate the association between personal behavior and lifestyle counseling has included only women physician providers (Franke et al., 2000) and did not include other healthcare providers such as nurse practitioners or physician assistants who provide a significant proportion of primary care in the US (Goolsby, 2003).

Therefore, our objective for this survey was to assess the impact of personal health habits of healthcare providers on the beliefs and practices of lifestyle counseling to elderly hypertensive patients in the US.

\section{Methods}

\section{Design and Sample}

This is a cross-sectional survey study to test the hypothesis that personal health habits of healthcare providers are associated with providers' beliefs and practices of lifestyle counseling to elderly hypertensive patients. For this survey, we defined a primary care provider as "a physician or nurse practitioner who has an active membership in the American Medical Association (AMA) or the American Academy of Nurse Practitioner (AANP)." We limited our selection criteria to those who have listed one of the following specialties: (1) general practice, (2) family medicine, (3) family practice, (4) geriatric medicine, (5) sports medicine, (6) internal medicine, and (7) primary care. There were 171,916 physicians listed in the 2005 AMA active membership directory and 10,225 nurse practitioners in AANP. To obtain a 3.5\% sampling error, we estimated a need for 781 returned surveys to obtain $95 \%$ confidence for our findings. We also anticipated an 8-12\% response rate, hence, we selected a random sample of 7,500 primary care providers. An anonymous survey and cover letter introducing the investigators and explaining the survey were mailed to each participant in our selected sample. The Institutional Review Board approved the protocol and the anonymous survey. Completion of the survey indicated written consent to participate in the study. Comparisons between responders and nonresponders and physicians and non-physicians could not be performed due to the anonymity of the survey.

\section{Lifestyle Counseling Theoretical Framework}

We developed a theoretical framework based on Lewis' Model for predicting the counseling practices of physicians (Lewis, Wells, \& Ware, 1986) and the theory of planned behavior (Godin \& Kok, 1996; Godin \& Shephard, 1990). In this model, providers' counseling is related to personal beliefs and attitudes about disease prevention and their own personal health habits. We maintained the same overall structure of the model and modified it to match our objective of hypertension specific counseling (Figure 1). 


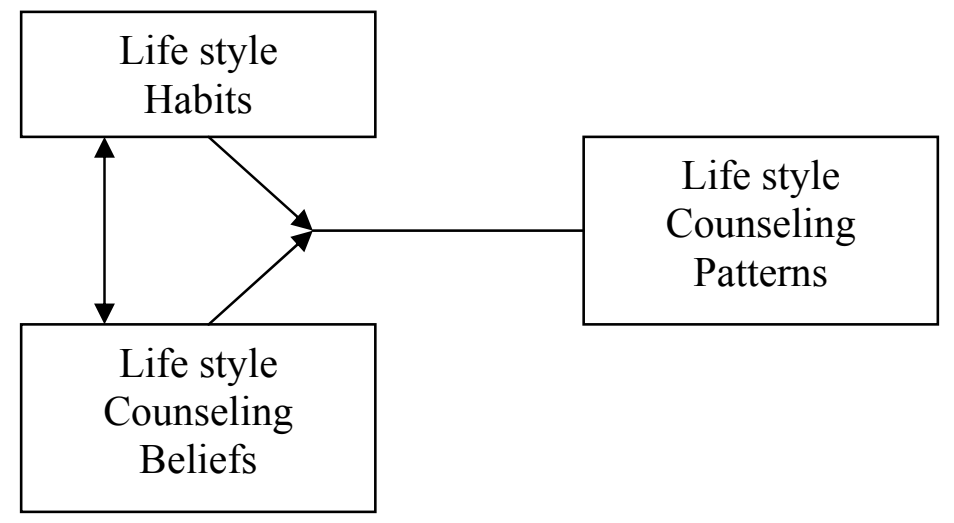

Figure 1

Modified Lewis' Model for predicting the counseling practices of physicians (Lewis, Wells, \& Ware, 1986) and the theory of planned behavior (Godin \& Kok, 1996; Godin \& Shephard, 1990)

Our model included lifestyle counseling patterns which reflect both obtaining a lifestyle history and providing lifestyle counseling to the elderly hypertensive patient. Lifestyle beliefs are defined as the cognitive opinion about lifestyle counseling. Lifestyle habits are defined as the activities that would impact the provider's health such as physical activity or dietary consumption of fruits and vegetables. The theoretical framework of this study proposes lifestyle habits of the primary care provider impacts both counseling beliefs and patterns, and counseling beliefs also impact counseling patterns. We intended to validate this framework using this survey.

\section{Instrument}

We developed a survey that measured the three aspects of our conceptual framework. Lifestyle counseling patterns that we assessed included: (1) obtaining a lifestyle history, and (2) providing lifestyle counseling that facilitates blood pressure control (hypertension specific) through improving weight, physical activity, diet, stress management, alcohol intake, and tobacco use cessation. Lifestyle counseling beliefs assessed included (1) the belief that the lifestyle counseling provided was effective in improving blood pressure control (effectiveness), (2) the belief that the provider had enough time to spend on lifestyle counseling during a usual visit (perceived time) and (3) the belief that the provider is skilled and trained in lifestyle counseling (perceived skill). The personal habits we assessed included (1) smoking status, (2) exercise frequency and duration, (3) consumption of fruits and vegetables, (4) consumption of low fat dairy, (5) consumption of alcohol, and (6) perceived stress level.

The instrument was developed using questions that have been validated in measuring the phenomenon in question. Questions from the Physician Health Survey (Christen, Gaziano, \& Hennekens, 2000) the National Health and Nutrition Examination Survey (NHANES) (Centers for Disease Control and Prevention, 1999-2000) and the Perceived Stress Scale (Hewitt, Flett, Mosher, 1992) were used. In the section of the survey asking about lifestyle counseling patterns, we used the scenario of usual visit of an elderly hypertensive patient to the provider. The participants were asked to report the frequency of the counseling belief or pattern using a Likert-like scale (never $=0$ to always $=4$ ).

\section{Confounders}

To identify potential confounders, we performed literature searches for factors that have been related to lifestyle counseling practices and 
beliefs. We selected factors and beliefs that are relevant to hypertension and that have been related to lifestyle counseling in the areas of weight control, physical activity, dietary habits, stress coping, and alcohol consumption. Our selected potential predictors of lifestyle counseling were: (1) provider's age, (2) gender, (3) specialty, (4) percentage of time spent in direct patient care, (5) location categorized as Northeast (NE), Midwest (MW), Southeast (SE), or West (W), (6) training in lifestyle counseling, and (7) provider's diagnosis of hypertension. We elected not to ask about providers' weight since in the Women's Physician Health Study (WPHS), weight or trying to lose weight was not related to lifestyle counseling (Frank et al., 2000). Wells et al. (1984) found that perceived weight was not related to primary prevention. In addition, a recent European survey did not find a relationship between physician reported weights or body mass index and counseling for weight loss (Kloppe et al., 2005).

\section{Statistical Analysis}

For the hypertension specific counseling patterns we calculated a composite score by adding the responses on the question of "I facilitate blood pressure control in elderly patients by providing effective counseling to: lower dietary salt (sodium), increase dietary potassium, increase physical activity, lose weight, stop smoking, reduce life stressors, and limit alcohol intake" (score $=0$ to 28). Higher score meant that the provider reported delivering lifestyle counseling more frequently. We also calculated a composite score for provider's lifestyle habits by adding the responses to smoking status, composite physical activity (PA) score (product of days of vigorous exercise per week and duration of average exercise session), servings of fruits and vegetables, low fat dairy, alcohol consumption, and stress level (0-30). Higher score meant healthier habits.

Our main outcome variables were lifestyle counseling patterns (the reported frequency of obtaining lifestyle history and providing hypertension specific counseling score). Our other outcomes were lifestyle counseling beliefs (reported frequency of counseling being effective and of having adequate time and skill to provide counseling). Our predictor was the lifestyle habit score. To assess the impact of health habits on counseling patterns and beliefs we used multiple regression analyses to adjust for confounding and results are displayed as regression coefficients $(\beta)$ and standard error (SE).

\section{Results}

Sample and Lifestyle Habits: We received 763 of the 7500 surveys. Characteristics of the respondents are shown in Table 1. A third of the providers reported they have never or rarely received training about lifestyle counseling. Due to the anonymous nature of this survey we were unable to compare responders and nonresponders.

Of those responding, 78\% have never smoked and $20 \%$ were past smokers. Less than a quarter $(23 \%)$ of providers reported they exercise five or more days a week and $69 \%$ exercise one to four days. Only $8 \%$ of the responding providers reported they consume more than six servings of fruits and vegetables, and $73 \%$ consume less than three servings of low fat dairy products a day. The majority $(90 \%)$ reported low level of stress and $83 \%$ consumed none to one alcoholic drink per day. The overall median providers' health habits score was 18 (range: 8-25).

\section{Beliefs}

\section{Lifestyle Counseling Patterns and}

Of those who responded to our survey, $89 \%$ reported they often or always obtain lifestyle history. When asked about hypertension-specific lifestyle counseling, the majority often or always counseled patients to lower salt $(80 \%)$, increase physical activity (92\%), lose weight (89\%), stop smoking $(95 \%)$, and limit alcohol $(83 \%)$, but only $34 \%$ recommended increasing potassium intake. The majority never or rarely counseled on reducing stress. The overall median lifestyle counseling score was 29 (range: 7-35). Of those who responded, $61 \%$ believed that they often or always provide effective lifestyle counseling, $55 \%$ have adequate time to spend on lifestyle counseling, and were skilled in providing lifestyle counseling to their elderly hypertensive patients. 
Table 1

Characteristics of healthcare providers who responded to our survey $(\mathrm{N}=763)$

\begin{tabular}{|l|l|}
\hline \multicolumn{1}{|c|}{ Characteristic } & \multicolumn{1}{|c|}{ Statistic (N=763) } \\
\hline Age in years, Mean \pm Standard Error (SE) & $49 \pm 0.4$ years \\
\hline Women, N (\%) & $442(58 \%)$ \\
\hline Location of practice, N (\%) & \\
\hline North & $198(26 \%)$ \\
\hline Midwest & $175(23 \%)$ \\
\hline West & $153(20 \%)$ \\
\hline South & $237(31 \%)$ \\
\hline Specialty, N (\%) & \\
\hline Internal Medicine & $221(21 \%)$ \\
\hline Family Medicine & $359(47 \%)$ \\
\hline Other specialty & $183(24 \%)$ \\
\hline Average time in direct patient care, \% \pm SE & $84 \pm 1 \%$ \\
\hline $\begin{array}{c}\text { Those who never or rarely received training during residency } \\
\text { or medical school on lifestyle counseling, N (\%) }\end{array}$ & $252(33 \%)$ \\
\hline Diagnosis of hypertension in healthcare providers, N (\%) & $153(20 \%)$ \\
\hline
\end{tabular}

Lifestyle habits and lifestyle counseling patterns: Providers who reported healthier lifestyle habits measured by the lifestyle habit score were more likely to report more frequent lifestyle counseling patterns in their elderly hypertensive patients including obtaining lifestyle history $(\mathrm{p}=.004)$ and delivering hypertension specific counseling $(\mathrm{p}<.001)$. When we assessed individual lifestyle habits, higher fruit and vegetable consumption and physical activity and lower stress levels but not low fat diary product consumption or alcohol intake were related to lifestyle counseling patterns (Table 2).
Lifestyle Habits and Lifestyle Counseling Beliefs. Similarly, those with healthier habits measured by the lifestyle habit score more frequently believed that they provided effective lifestyle counseling to help control hypertension in elderly patients $(\mathrm{p}<.001)$, that they had adequate time available to spend on lifestyle counseling $(\mathrm{p}=.008)$ and that they were skilled in lifestyle counseling $(\mathrm{p}<.001)$. Only fruit and vegetable consumption and physical activity were associated with the counseling beliefs (Table 3).

Table 2

Associations between reported lifestyle habits of health care providers and counseling patterns for elderly hypertensive patients $(\mathrm{N}=763)$

\begin{tabular}{|c|c|c|c|c|}
\hline Provider lifestyle habit & $\begin{array}{c}\text { Obtaining } \\
\text { lifestyle history } \\
\beta(\mathrm{SE}) \\
\end{array}$ & $\mathbf{p}$ & $\begin{array}{c}\text { HTN-specific } \\
\text { counseling } \\
\beta(\mathrm{SE})\end{array}$ & $\mathbf{p}$ \\
\hline Physical activity & $0.03(0.01)$ & .009 & $0.2(0.06)$ & .001 \\
\hline Daily fruits and vegetable servings & $0.1(0.04)$ & .001 & $0.8(0.2)$ & .001 \\
\hline Daily low fat dairy servings & $-0.0001(0.03)$ & .986 & $0.2(0.2)$ & .493 \\
\hline Daily alcohol consumption & $0.04(0.03)$ & .19 & $-0.2(0.2)$ & .323 \\
\hline Monthly episodes of stress & $-0.08(0.03)$ & .034 & $0.5(0.2)$ & .027 \\
\hline Overall health habits score & $0.02(0.01)$ & .004 & $0.2(0.1)$ & $<.001$ \\
\hline
\end{tabular}


Table 3

Associations between reported lifestyle habits of health care providers and providers' beliefs about lifestyle counseling for elderly hypertensive patients $(\mathrm{N}=763)$

\begin{tabular}{|l|l|l|c|c|c|c|}
\hline \multicolumn{1}{|c|}{ Provider lifestyle habit } & $\begin{array}{c}\text { Effectiveness } \\
\boldsymbol{\beta} \text { (SE) }\end{array}$ & $\mathbf{p}$ & $\begin{array}{c}\text { Perceived } \\
\text { Time } \\
\boldsymbol{\beta} \text { (SE) }\end{array}$ & $\mathbf{p}$ & $\begin{array}{c}\text { Perceived } \\
\text { skills } \\
\boldsymbol{\beta} \text { (SE) }\end{array}$ & $\mathbf{p}$ \\
\hline Physical activity & $0.04(0.01)$ & .002 & $0.03(0.01)$ & .009 & $0.06(0.01)$ & $<.001$ \\
\hline Daily fruits and vegetable servings & $0.2(0.05)$ & .001 & $0.1(0.05)$ & .011 & $0.3(0.05)$ & $<.001$ \\
\hline Daily low fat dairy servings & $0.09(0.04)$ & .44 & $-0.02(0.04)$ & .628 & $0.07(0.04)$ & .11 \\
\hline Daily alcohol consumption & $0.07(0.04)$ & .111 & $0.04(0.04)$ & $<.001$ & $0.09(0.04)$ & .021 \\
\hline Monthly episodes of stress & $0.1(0.05)$ & .005 & $0.08(0.04)$ & $<.056$ & $0.07(0.4)$ & .106 \\
\hline Overall health habits score & $0.05(0.01)$ & $<.001$ & $0.03(0.01)$ & .008 & $0.06(0.01)$ & $<.001$ \\
\hline
\end{tabular}

$\mathrm{SE}=$ Standard Error. The coefficients $(\beta)$ were obtained from regression analysis of overall lifestyle counseling beliefs as the dependent variable and the various lifestyle habits as the independent variable.

Lifestyle Counseling Beliefs and Patterns. As we expected those who had positive beliefs about lifestyle counseling were more likely to report more frequent lifestyle counseling. In particular, those who felt they had more time to spend on lifestyle counseling $(\beta=3.0 \quad(0.1)$; $\mathrm{p}<.001)$, those who felt they were skilled in lifestyle counseling $(\beta=2.3(0.2) ; p<.001)$, and those who believed that lifestyle counseling was effective $(\beta=1.9(0.2), p<.001)$ were more likely to report more frequent lifestyle counseling patterns for elderly hypertensive patients.

Multivariate analysis. After adjusting for potential confounders (provider's age, gender, location, specialty, and previous training in counseling) health habits were consistently associated with lifestyle counseling patterns $(p=.03$ for lifestyle history and $p<.001$ for hypertension specific counseling) and beliefs $(p<.001$ for effectiveness and perceived skills and $\mathrm{p}=.038$ for perceived time).

\section{Discussion and Conclusion}

This survey suggests that primary care providers with healthier lifestyle habits more frequently counseled their elderly hypertensive patients on better lifestyle and had positive beliefs about counseling. Specifically, providers who exercised more, consumed more fruits and vegetables, and experienced less stress, were more likely to report more frequent lifestyle counseling. It also confirmed our conceptual framework of the interaction between lifestyle habits and counseling beliefs and practices.
To our knowledge, this is the first national survey that assesses the association between providers' lifestyle behaviors and their beliefs and practices of providing counseling to their elderly hypertensive patients. The association between providers' habits and general counseling have been reported previously in women physicians (Frank, Bhat Schelbert, \& Elon, 2003; Frank et al., 2000). We have identified a similar association with counseling practices for hypertensive elderly individuals. In our survey, there was no difference by gender suggesting that these associations are applicable to men and women providers.

Our theoretical framework identifies a potential mechanism to explain why providers with healthier lifestyle are more likely to provide frequent counseling. The impact of lifestyle habits on beliefs about lifestyle counseling may explain this association. Altering providers' beliefs about counseling through training or education may be an accessible modifiable target for interventions that aim to improve lifestyle counseling in primary care. The effectiveness of such an intervention needs to be confirmed with a randomized clinical trial. In addition, we have identified a relationship between providers' levels of stress and their frequency of lifestyle counseling. This is to our knowledge a new finding and opens a new avenue for possible improvement in lifestyle counseling. 
Both the Centers for Disease Control and Prevention and the American College of Sports Medicine recommend accumulating at least 30 minutes or more of moderate physical activity (Pate et al., 1995). Less than a quarter of our respondents perform physical activity five or more days, and most spend less than 30 minutes. This is lower than the recently reported general population rate of $45 \%$ (Centers for Disease Control and Prevention, 2005). Moreover, dietary guidelines for healthy living recommend consuming more than five servings of fruit and vegetables per day (Kuczmarski \& Weddle, 2005). Less than $10 \%$ of our sample was in compliance with this recommendation. Taken together, healthcare providers are leading an unhealthy lifestyle from a nutritional and physical activity perspective. This suggests that there is considerable opportunity to improve providers' lifestyle habits which may have an impact on their beliefs and practices of providing lifestyle counseling to their hypertensive patients.

A main limitation of our survey is that our sample is not highly representative of the population studied. We anticipated a low response rate, thus, 7500 surveys were mailed, leading to a $3.5 \%$ sampling error. Therefore, although the hypothesis was validated with this survey, response bias should be considered when interpreting these results. Due to the anonymity of this survey we were unable to compare responders with non-responders. It is possible that those who responded were more likely to provide counseling or more likely to have healthier habits. Few of our responding providers reported they exercise more than five times per week and consume more than six servings of fruit and vegetables suggesting suboptimal lifestyle behaviors. We were also unable to compare different types of providers.

The advantages of this survey is that it included both women and men providers, both physicians and non-physicians, assessed both reported lifestyle practices and beliefs, and addresses counseling for a highly prevalent and potentially devastating illness in elderly individuals.

Providers' healthier lifestyle habits are associated with more positive beliefs and attitudes towards lifestyle counseling and lifestyle counseling practices in elderly hypertensive patients. Programs that would improve both beliefs and lifestyle habits in primary care providers may improve lifestyle counseling to elderly hypertensive patients, which in turn may improve the control rates in this population.

\section{References}

Abramson, S., Stein, J., Schaufele, M., Frates, E., \& Rogan, S. (2000). Personal exercise habits and counseling practices of primary care physicians: a national survey. Clinical Journal of Sport Medicine, 10(1), 40-48.

Calfas, K. J., Long, B. J., Sallis, J. F., Wooten, W. J., Pratt, M., \& Patrick, K. (1996). A controlled trial of physician counseling to promote the adoption of physical activity. Preventive Medicine, 25, $225-$ 233.

Centers for Disease Control and Prevention (CDC \& P). (2005). Adult participation in recommended levels of physical activity - United States, 2001 and 2003. Morbidity and Mortality Weekly Report, 54, 1208-1212.

Centers for Disease Control and Prevention (1999-2000). NHANES 1999-2000 Public data release file documentation. Retrieved December 31, 2006, from http://www.cdc.gov/nchs/data/nhanes/gendoc.pdf

Centers for Disease Control and Prevention (CDC \& P). (1999). Physician advice and individual behaviors about cardiovascular disease risk reduction- seven states and Puerto Rico, 1997. Morbidity and Mortality Weekly Report, 48, 74-77.

Chobanian, A. V., Bakris, G. L., Black, H. R., Cushman, W. C., Green, L. A., Izzo, J. L., Jr., et al. (2003). Seventh report of the Joint National Committee on Prevention, Detection, Evaluation, and Treatment of High Blood Pressure. Hypertension, 42, 1206-1252. 
Christen, W. G., Gaziano, J. M., \& Hennekens, C. H. (2000). Design of Physicians' Health Study II--a randomized trial of beta-carotene, vitamins $\mathrm{E}$ and $\mathrm{C}$, and multivitamins, in prevention of cancer, cardiovascular disease, and eye disease, and review of results of completed trials. Annals of Epidemiology, 10(2), 125-134.

Collins, R. L., D'Angelo, S., Stearns, S. D., \& Campbell, L. R. (2005). Training pediatric residents to provide smoking cessation counseling to parents. ScientificWorld Journal, 5, 410-419.

Egede, L. E., \& Zheng, D. (2002). Modifiable cardiovascular risk factors in adults with diabetes: prevalence and missed opportunities for physician counseling. Archives of Internal Medicine, $162,427-433$.

Fallon, E. A., Wilcox, S., \& Laken, M. (2006). Health care provider advice for African American adults not meeting health behavior recommendations. Preventing Chronic Disease, 3(2), A45. Retrieved December 31, 2006, from http://www.cdc.gov/pcd/issues/2006/apr/pdf/05 0103.pdf

Frank, E., Bhat Schelbert, K., \& Elon, L. (2003). Exercise counseling and personal exercise habits of US women physicians. Journal of the American Medical Women's Association, 58(3), 178-184.

Frank, E., Breyan, J., \& Elon, L. (2000). Physician disclosure of healthy personal behaviors improves credibility and ability to motivate. Archives of Family Medicine, 9(3), 287-290.

Godin, G., \& Kok, G. (1996). The theory of planned behavior: a review of its applications to healthrelated behaviors. American Journal of Health Promotion, 11(2), 87-98.

Godin, G., \& Shephard, R. J. (1990). Use of attitude-behaviour models in exercise promotion. Sports Medicine, 10(2), 103-121.

Goolsby, M. J. (2003). 2001-2002 AANP national nurse practitioner practice site survey. Journal of the American Academy of Nurse Practitioners, 15(11), 482-484.

Greenlund, K. J., Giles, W. H., Keenan, N. L., Croft, J. B., \& Mensah, G. A. (2002). Physician advice, patient actions, and health-related quality of life in secondary prevention of stroke through diet and exercise. Stroke, 33, 565-570.

Hajjar, I., Miller, K., \& Hirth, V. (2002). Age-related bias in the management of hypertension: a national survey of physicians' opinions on hypertension in elderly adults. Journal of Gerontology Series A Medical Sciences, 57, M487-491.

Hansson, L., Hedner, T., \& Himmelmann, A. (1999). The 1999 WHO-ISH Guidelines for the Management of Hypertension--new targets, new treatment and a comprehensive approach to total cardiovascular risk reduction. Blood Pressure, 1(Suppl.), 3-5.

Hewitt, P., Flett, G., Mosher, S. (1992). The perceived stress scale: Factor structure and relation to depression symptoms in a psychiatric sample. Journal of Psychopathology and Behavioral Assessment, 14, 247-257.

Kloppe, P., Brotons, C., Anton, J. J., Ciurana, R., Iglesias, M., Pineiro, R., et al. (2005). Preventive care and health promotion in primary care: Comparison between the views of Spanish and European doctors. Atención Primaria, 36(3), 144-151.

Kuczmarski, M. F., \& Weddle, D. O. (2005). Position paper of the American Dietetic Association: nutrition across the spectrum of aging. Journal of the American Dietetic Association, 105, 616633.

Lewis, C. E., Wells, K. B., \& Ware, J. (1986). A model for predicting the counseling practices of physicians. Journal of General Internal Medicine, 1(1), 14-19.

Linden, W., Lenz, J. W., \& Con, A. H. (2001). Individualized stress management for primary hypertension: a randomized trial. Archives of Internal Medicine, 161, 1071-1080.

Litaker, D., Flocke, S. A., Frolkis, J. P., \& Stange, K. C. (2005). Physicians' attitudes and preventive care delivery: Insights from the DOPC study. Preventive Medicine, 40, 556-563.

Ma, J., Urizar, G. G., Jr., Alehegn, T., \& Stafford, R. S. (2004). Diet and physical activity counseling during ambulatory care visits in the United States. Preventive Medicine, 39, 815-822.

Ockene, I. S., Hebert, J. R., Ockene, J. K., Saperia, G. M., Stanek, E., Nicolosi, R., et al. (1999). Effect of physician-delivered nutrition counseling training and an office-support program on saturated fat 
intake, weight, and serum lipid measurements in a hyperlipidemic population: Worcester area trial for counseling in hyperlipidemia (WATCH). Archives of Internal Medicine, 159, 725-731.

Pate, R. R., Pratt, M., Blair, S. N., Haskell, W. L., Macera, C. A., Bouchard, C., et al. (1995). Physical activity and public health. A recommendation from the Centers for Disease Control and Prevention and the American College of Sports Medicine. Journal of the American Medical Association, 273, 402-407.

Petrella, R. J., \& Lattanzio, C. N. (2002). Does counseling help patients get active? Systematic review of the literature. Canadian Family Physician, 48, 72-80.

Shah, S., \& Cook, D. G. (2001). Inequalities in the treatment and control of hypertension: age, social isolation and lifestyle are more important than economic circumstances. Journal of Hypertension, $19,1333-1340$.

Wells, K. B., Lewis, C. E., Leake, B., \& Ware, J. E., Jr. (1984). Do physicians preach what they practice? A study of physicians' health habits and counseling practices. Journal of the American Medical Association, 252, 2846-2848.

\author{
Author Information \\ Ihab M. Hajjar, MD, MS, Associate Director* \\ Cardiovascular Research Lab \\ Hebrew SeniorLife \\ Instructor of Medicine; Harvard Medical School \\ 1200 Centre Street \\ Boston, MA 02132 \\ Ph.: 617-363-8179 \\ Fax.: 617-363-8936 \\ Brandy Dickson, ND \\ Assistant Clinical Professor for Internal Medicine \\ Division of Geriatrics \\ Center for Senior Hypertension \\ Palmetto Health Richland \\ University of South Carolina, School of Medicine \\ 3010 Farrow Road; Suite 300 \\ Columbia, SC 29203 \\ $\mathrm{Ph} .:$ 803-434-2595 \\ Fax.: 803-434-4331 \\ E-Mail: brandy.frost@palmettohealth.org \\ John E. Lacy, BS, Medical Student \\ University of South Carolina, School of Medicine \\ Columbia, SC 29208 \\ Jane Morley Kotchen, MD, MPH, Professor \\ Division of Epidemiology \\ Department of Population Health \\ Medical College of Wisconsin \\ 8701 Watertown Plank Road \\ Milwaukee, WI 53226 \\ * corresponding author
}

